\title{
Detection of brucellosis through active surveillance, Armenia, 2014
}

\author{
Liana Torosyan*, Lilit Avetisyan and Artavazd Vanyan \\ National Center for Disease Control and Prevention, Ministry of Health, Republic of Armenia, Yerevan, Armenia
}

Objective

In the spring of 2014, people from vulnerable households in all marzes of Armenia were examined with the aim of active surveillance.

\section{Introduction}

Brucellosis is a serious disease caused by bacteria of the Brucella genus. It principally affects ruminants but may be transmitted to humans. Registration of cases in cattle farms causes considerable economic losses and creates favorable conditions for mass infection among humans. In Armenia the expansion of animal industries and urbanization are the main reasons for occurrence and development of brucellosis.

\section{Methods}

Blood was sampled from people on farms reported as having infected animals. Blood samples were tested by the WrightHuddleston method. The standard case definition of brucellosis was used for diagnosis. A questionnaire-based interview was carried out among the population to identify the form of contact with animals and to analyze epidemiological links. During the investigation provisions were followed in governmental decree RA 19.01.2006 N480-N and brucellosis prevention, epidemiology, diagnosis, treatment, preventive measures.

\section{Results}

A total of 11160 people from 1054 households were enrolled in the study, of which 3625 (32.5\%) underwent a laboratory examination. Nearly $6 \%$ (641) refused to be tested. Over $6 \%$ of those tested (226) were positive for antibodies to Brucellae. Of these, 129 (3.5\%) had chronic brucellosis. Those testing positive for brucellosis were treated appropriately. These included 203 (90\%) adults and $23(10 \%)$ below 14 years old; $147(65 \%)$ were male and 79 (35\%) were female. Of those diagnosed with brucellosis, working in animal husbandry accounted for $46.6 \%$ (106), while those who harvested milk accounted for $37.6 \%$ (85) and those using raw milk made up $15.4 \%$ (35).

\section{Conclusions}

Cases were most frequently reported among people 20-55 years of age; the highest percentage of positives were among 41-45 year old males who had contact with infected animals. The main risk factor for acquiring brucellosis is animal husbandry.

\section{Keywords}

brucellosis; surveillance; Armenia

\author{
*Liana Torosyan \\ E-mail: Liana_torosyan@mail.ru
}

